\title{
Superconcentrated electrolytes widens insertion electrochemistry to soluble layered halides
}

Nicolas Dubouis ${ }^{1,2,3,5}$, Thomas Marchandier ${ }^{1,2,3,5}$, Gwenaelle Rousse ${ }^{1,2,3}$, Florencia Marchini ${ }^{1,2,3}$, François Fauth4, Maxim Avdeev ${ }^{5,6}$, Antonella ladecola ${ }^{3}$, Benjamin Porcheron $^{3,7}$, Michael Deschamps $^{3,7}$, Jean-Marie Tarascon ${ }^{1,2,3, *}$ and Alexis Grimaud ${ }^{1,2,3, *}$

\section{\$: Equal contribution}

*Corresponding authors: alexis.grimaud@college-de-france.fr, jean-marie.tarascon@college-de-

$\underline{\text { france.fr }}$

1. Collège de France, Chaire de Chimie du Solide et de I'Energie, UMR 8260, 11 place Marcelin Berthelot, 75231 Paris Cedex 05, France

2. Sorbonne Université- 4 place Jussieu, F-75005 Paris, France

3. Réseau sur le Stockage Electrochimique de I'Energie (RS2E), FR CNRS 3459, 80039 Amiens, France

4. CELLS -ALBA synchrotron, Cerdanyola del Valles, Barcelona E-08290, Spain

5. School of Chemistry, The University of Sydney, Sydney, NSW 2006, Australia

6. Australian Centre for Neutron Scattering, Australian Nuclear Science and Technology Organisation, New Illawarra Rd, Lucas Heights, NSW 2234, Australia

7. CNRS-CEMHTI, UPR3079, Université d'Orléans, 1D avenue de la recherche scientifique, 45071 Orléans Cedex, France 


\begin{abstract}
Insertion compounds provide the fundamental basis of today's commercialized Li-ion batteries. Throughout history, intense research has focus on the design of stellar electrodes mainly relying on layered oxides or sulfides, and leaving aside the corresponding halides because of solubility issues. This is no longer true. In this work, we show for the first time the feasibility to reversibly intercalate electrochemically $\mathrm{Li}^{+}$into $\mathrm{VX}_{3}$ compounds ( $\mathrm{X}=\mathrm{Cl}, \mathrm{Br}, \mathrm{I})$ via the use of superconcentrated electrolytes, (5 M LiFSI in dimethyl carbonate), hence opening access to a novel family of $\mathrm{Li}_{x} V X_{3}$ phases. Moreover, through an electrolyte engineering approach we unambiguously prove that the positive attribute of superconcentrated electrolytes against solubility of inorganic compounds is rooted in a thermodynamic rather than a kinetic effect. The mechanism and corresponding impact of our findings enrich the fundamental understanding of superconcentrated electrolytes and constitute a crucial step in the design of novel insertion compounds with tunable properties for a wide range of applications beyond Li-ion batteries.
\end{abstract}




\section{Introduction}

Redox chemistry provides the fundamental basis for numerous energy-related electrochemical devices, among which Li-ion batteries (LIB) have become the premier energy storage technology for portable electronics and vehicle electrification. Throughout its history, LIB research has witnessed a frenetic race for designing new intercalation compounds, so that most of the crystallographic families with open framework have been investigated and though chances to discover new promising phases are slim. Nevertheless, some compounds sharing similar structures with those of archetypal LIBs cathode materials have never been envisioned as potential host materials. For instance, while transition metals oxides and sulfides have been widely studied, members of the vanadium tri-halide family $V X_{3}(X=C l, B r$ or I) were never investigated as battery intercalation compounds despite sharing similar structure with the iconic Li-ion cathode layered materials $\mathrm{TiS}_{2}$ and $\mathrm{LiCOO}_{2}$. Yet, recent studies highlight the interesting physical properties of theses vanadium halide phases. ${ }^{1,2}$ For instance their magnetic and electronic structures can be finely tuned by playing with the interlayer coupling between the Van der Walls gap. ${ }^{3}$ Such structural modifications are easily achieved through cation intercalation, ${ }^{4}$ reinforcing our motivation for testing these materials as Li intercalation hosts. However, the absence of reports on layered halides intercalation compounds is partially explained by their high solubility in polar solvents, as highlighted by their use as vanadium precursors in redox-flow batteries. ${ }^{5}$

The delicate balance between materials and liquid electrolytes was already one of the main hurdles that delayed the commercialization of secondary lithium-ion batteries relying on insertion reactions. For instance, the physical and chemical integrity of carbonaceous electrodes were found altered upon lithium intercalation in polypropylene carbonate (PC) based electrolytes initially employed. ${ }^{6}$ Such difficulty was later on addressed by tuning the lithium solvation shell with the use of ethylene carbonate (EC), suppressing the co-intercalation of PC and forming a stable passivating layer on the carbon anode, ${ }^{7}$ or more recently by using superconcentrated electrolytes. ${ }^{8-10}$ In turn, history teaches us that an electrolyte engineering approach can be used to unlock lithium intercalation in phases hitherto believed to be too unstable for the application. 
Besides graphite intercalation, superconcentrated electrolytes have also shown promising functionalities to solve many fundamental drawbacks relative to the use of carbonate based electrolytes, such as incompatibility with Li-metal, instability at high voltages, flammability or dissolution of transition metals from the cathode. ${ }^{11-18}$ Hence, the use of superconcentrated electrolytes was identified as an attractive direction to explore the reversible intercalation of $\mathrm{Li}^{+}$into halide-based compounds such as the members of the $\mathrm{VX}_{3}$ family.

Herein we demonstrate the feasibility, via the use of superconcentrated electrolytes, to unlock the reversible electrochemical intercalation of lithium in vanadium halides previously considered as transition metal salts too soluble to be used in Li batteries. As a proof of concept, we studied the electrochemical behavior of three vanadium-based halides: $\mathrm{VCl}_{3}, \mathrm{VBr}_{3}$ and $\mathrm{VI}_{3}$. Combining electrochemical measurements with structural (synchrotron X-ray and neutron diffraction) and solubility measurements, we successfully demonstrate that nearly one $\mathrm{Li}^{+}$per formula unit can be reversibly intercalated into these materials, hence opening the door to new intercalation chemistries going beyond our current knowledge regarding oxides, sulfides or polyanionic compounds.

\section{Results}

\section{Intercalation of lithium into vanadium halides enabled thanks to superconcentrated}

\section{electrolytes}

While $\mathrm{VCl}_{3}$ is commercially available, $\mathrm{Vl}_{3}$ and $\mathrm{VBr}_{3}$ were grown in evacuated quartz sealed tubes by reacting elemental vanadium, iodine and bromine in nearly stoichiometric conditions (see methods). Their structure was found to match those reported in the literature, where edge-shared VX $\mathrm{X}_{6}$ octahedra form honeycomb layers stacked along the c direction with a $A B$ sequence (O1 type structure ${ }^{19}$ ) (Figure 1. a, Supplementary Figure 1, Supplementary Tables 1 and $\mathbf{2}$ ). These materials were tested by assembling battery half-cells to investigate their ability to electrochemical insert lithium cations $\left(\mathrm{Li}^{+}\right)$, using state-of-the-art LIB electrolyte 
LP30 (1 M of lithium hexafluorophosphate in an equivolume mixture of dimethyl carbonate, DMC) (Figure

1. b).

a

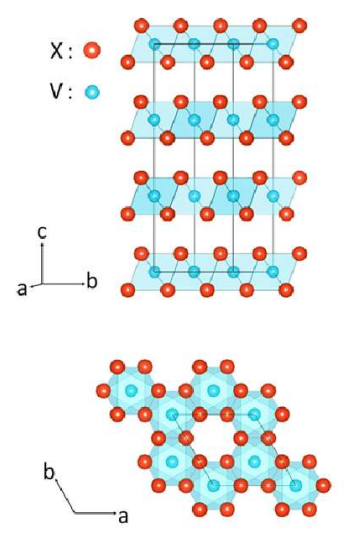

b

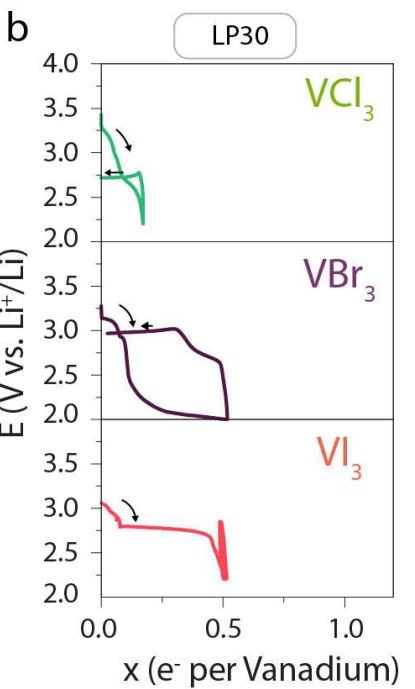

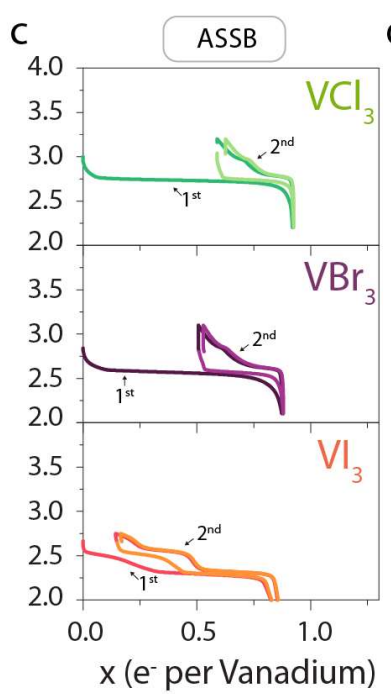

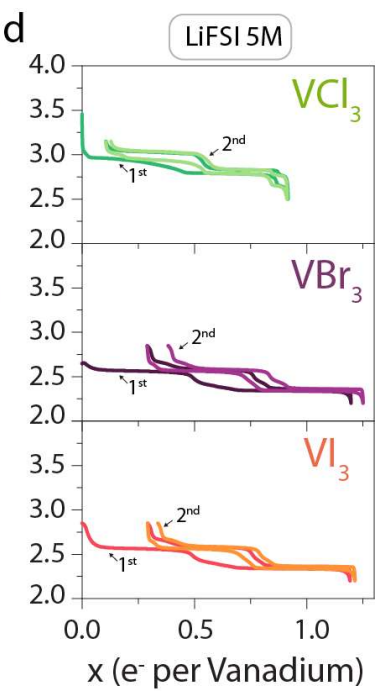

Figure 1. Electrolyte engineering to unlock reversible $\mathrm{Li}^{+}$intercalation in $\mathrm{VX}_{3}$ phases. a) Crystalline structure of $\mathrm{VX} \mathrm{X}_{3}(\mathrm{~V}$ atoms are in blue and $\mathrm{X}$ in orange, $\mathrm{X}=\mathrm{Cl}, \mathrm{Br}$ or I ), b) galvanostatic cycling of $\mathrm{VCl}_{3}$ (green), $\mathrm{VBr}_{3}$ (purple) and $\mathrm{Vl}_{3}$ (red) at $\mathrm{C} / 40$ using $\mathrm{LP} 30$ electrolyte and a Li(Metal) negative electrode, c) galvanostatic cycling of $\mathrm{VCl}_{3}$ (green), $\mathrm{VBr}_{3}$ (purple) and $\mathrm{Vl}_{3}$ (red) at $\mathrm{C} / 10$ in a solid-state configuration using argyrodite electrolyte and InLi negative electrode and d) galvanostatic cycling of $\mathrm{VCl}_{3}$ (green), $\mathrm{VBr}_{3}$ (purple) and $\mathrm{Vl}_{3}$ (red) at C/40 using a $5 \mathrm{M} \mathrm{LiFSI} \mathrm{in} \mathrm{DMC} \mathrm{electrolyte} \mathrm{and} \mathrm{a} \mathrm{Li(M)} \mathrm{negative} \mathrm{electrode.} \mathrm{For} \mathrm{C}$ ) and d) the first two cycles are plotted.

For these three compounds (Figure $\mathbf{1 ,} \mathbf{b}$ ), a relatively small discharge capacity is obtained and the process is clearly irreversible as no capacity is observed during the subsequent charge. Moreover, large amount of vanadium traces are observed after discharge on the negative electrode (Supplementary Figure 2), pointing to the dissolution of the cathode material and an irreversible solution process as the origin for the limited discharge capacity. Similar poor performances were encountered using ionic liquid electrolytes (Supplementary Figure 3). Hence, there is a need to develop a new strategy to overcome these dissolution issues. The first attempt consists in replacing the liquid electrolyte by a solid-state electrolyte in which no dissolution can occur. Effectively, when composite cathodes are prepared and tested in an all-solid-state battery configuration (see methods), the electrochemistry is greatly improved compared to that in LP30, thus hinting towards the electrochemical intercalation of $\mathrm{Li}^{+}$into $\mathrm{VX}_{3}$ compounds (Figure 1. c). However, in 
such configuration the complex interplay between ionic conductivity of the solid electrolyte, chemical compatibility between components and electrode microstructure requires a separate optimization of electrode formulation and testing conditions for each compound. This is indeed exemplified in the chemical incompatibility found between $\mathrm{VBr}_{3}$ or $\mathrm{Vl}_{3}$ and argyrodite solid-state electrolyte, evidenced as a clear color change-upon mixing. This observation, combined with the difficulty of assembling ASSB and characterizing active materials in such configuration, renders critical the search for an adequate liquid electrolyte in which halides would be stable.

To select the correct electrolyte, reconsidering the physico-chemical properties of classical aprotic Li-ion batteries electrolytes proves to be insightful. Commercial LP30 electrolyte is made from a mixture of carbonate solvents (EC and DMC) in which a salt $\left(\mathrm{LiPF}_{6}\right)$ is dissolved. While EC is a polar component essential to ensure both ion-pairs dissociation and the formation of a stable solid electrolyte interphase (SEI) at the negative electrode, its high dielectric constant is presumably responsible for the $\mathrm{VX}_{3}$ dissolution. Hence, not only the chemical composition of the electrolyte must be modified to prevent vanadium cations and/or halides solubility but also its solvation properties. Inspired by the recent observation of the non-miscibility of lithium halides salts with lithium imide salts, ${ }^{20,21}$ we then investigated the electrochemical behavior of vanadium halides in superconcentrated electrolytes. As shown in Figure $\mathbf{1} \mathbf{d}$, when LP30 is substituted with a $5 \mathrm{M}$ lithium bis(fluorosulfonyl)imide (LiFSI) in DMC electrolyte (denoted LiFSI $5 \mathrm{M}$ hereafter), the electrochemical behavior of the three different phases is drastically even at C-rates as low as C/40, indicating that superconcentrated electrolytes can tackle the solubility issue observed in LP30. Indeed, albeit some initial irreversible capacity being observed in the first cycle, these results suggest that almost $1 \mathrm{Li}^{+}$per formula unit can be reversibly inserted into these hosts. Furthermore, no vanadium was found at the negative electrode at the end of discharge (Supplementary Figure 2). Overall, switching from regular diluted carbonate-based electrolytes to a superconcentrated electrolyte appears as a key to unlock the electrochemical intercalation of $\mathrm{Li}^{+}$into halide layered compounds. 
a

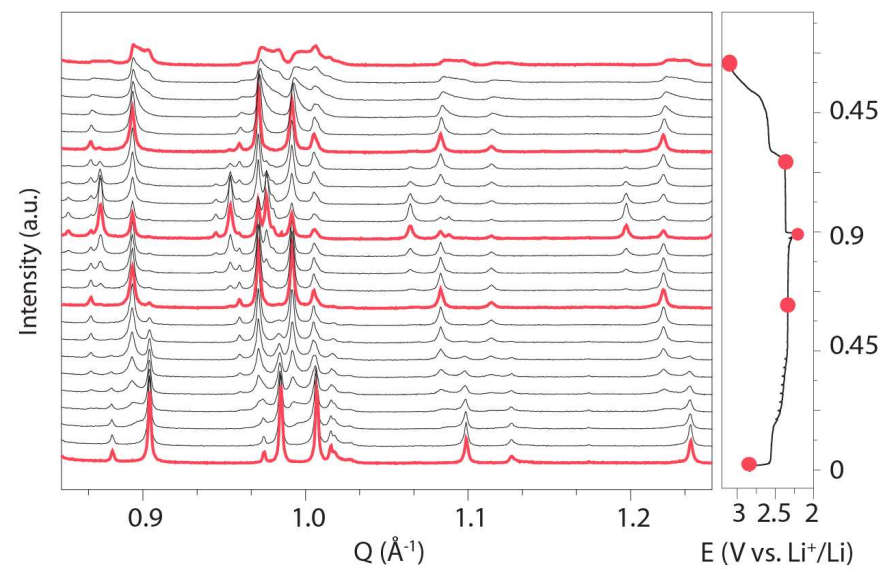

b

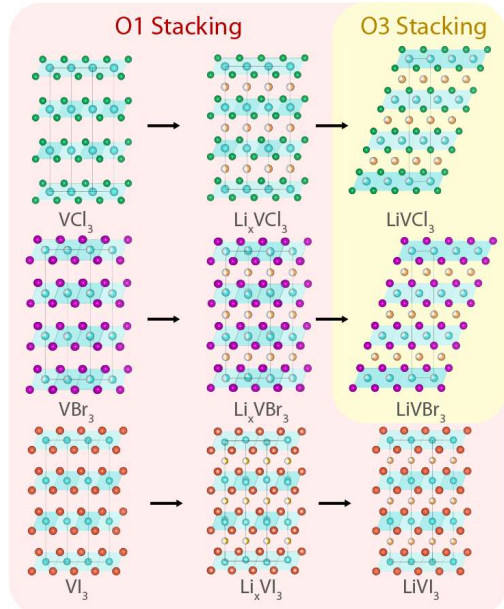

Figure 2. Evolution of the material crystalline structure upon cycling. a) Operando synchrotron $\mathrm{X}$-ray diffraction of $\mathrm{V} \mathrm{l}_{3}$ upon cycling shows a reversible phase transformation with the growth of an intermediate and end of discharge phases and $\mathbf{b}$ ) crystalline structures of pristine, intermediate and end of discharge phases for $\mathrm{VCl}_{3}, \mathrm{VBr}_{3}$ and $\mathrm{Vl}_{3}$

To grasp further insights into the behavior upon cycling of these halides, their structural evolution was monitored by operando synchrotron X-ray diffraction (SXRD). For $L \mathrm{i}_{\times} \mathrm{VI}_{3}$ (Figure $\mathbf{2}$ a), two successive biphasic processes can be distinguished upon discharge, consistent with the two plateaus previously observed (Figure $1 \mathbf{d}$ ). For $0<x<0.6$, the pristine phase disappears at the expense of an intermediate phase which is then replaced by a fully lithiated phase. On charge, the process is found reversible with the discharged phase being converted back to the pristine one; similar reversible bi-phasic intercalation processes are observed for both $\mathrm{VCl}_{3}$ and $\mathrm{VBr}_{3}$ (Supplementary Figures 4 and 5). Having proved the full structural reversibility of the insertion process and the absence of vanadium dissolution, we believe that part of the observed irreversible capacity is mostly nested in minute amounts of amorphous impurities of chemically absorbed I or $\mathrm{Br}$ in our starting materials that were made by gas phase reactions. The structure of every intermediate and fully lithiated phases were then determined by Rietveld refinement. This analysis reveals that the O1type layered structure ( $R \overline{3}$ space group) of the pristine is preserved for every intermediate phase, with the sole evolution of the lattice parameters (Supplementary Figure 6 and Supplementary Tables 3 and 4). Unlike for the intermediate phases, the structure of the fully discharged phases is dependent on the nature of the anion. Hence, while for $\mathrm{Vl}_{3}$ the fully discharged phase possesses the $\mathrm{O} 1$ structure, for $\mathrm{VCl}_{3}$ and $\mathrm{VBr}_{3}$ a 
distortion to an $\mathrm{O} 3$ layered structure $(R \overline{3} m$ space group) is observed (Supplementary Figures $\mathbf{7}$ and $\mathbf{8}$,

Supplementary Tables 5,6 and 7), in agreement with a previous report on a $\mathrm{VCl}_{3}$ lithiated phase prepared by a solid-state route. ${ }^{22}$ Such a subtle Li-driven structural difference depending upon the nature of the halide may simply be rooted in their size, following the ionic radii $\mathrm{Cl}<\mathrm{Br}<\mathrm{I}$. Finally, and to no surprise for layered compounds, it was confirmed by neutron powder diffraction experiments that lithium cations sit in the interlayer of the phases (Supplementary Figure 7).

a

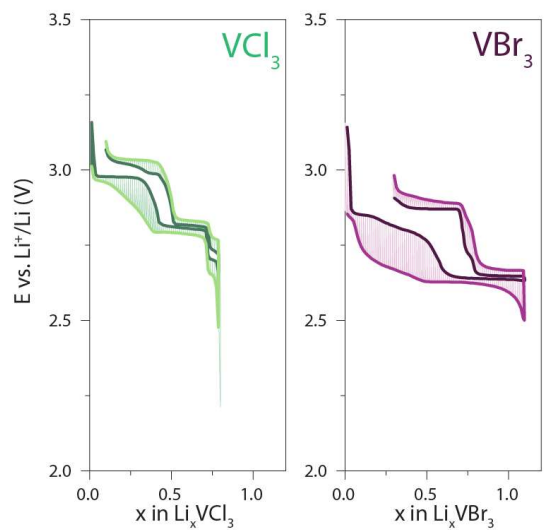

C

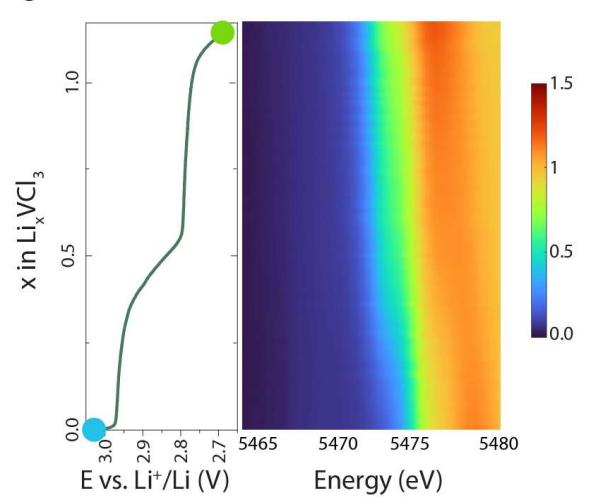

b

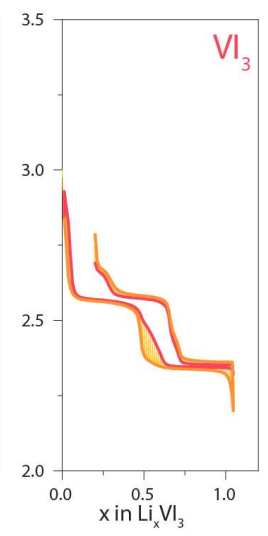

d

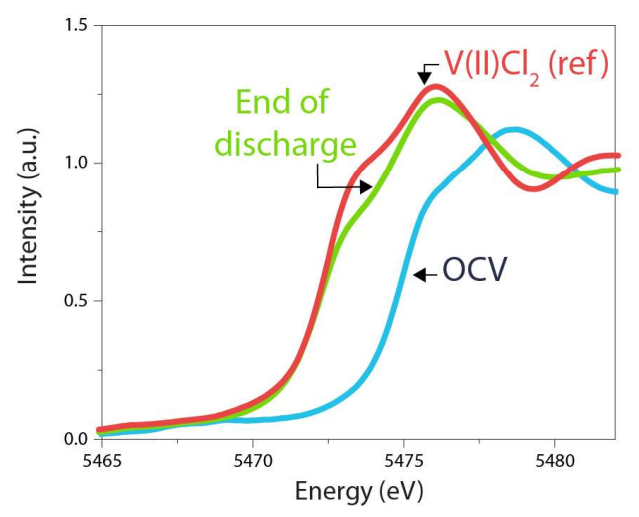

Figure 3. Determination of the charge compensation mechanism a) Galvanostatic intermittent titration technique measurements of $\mathrm{VCl}_{3}$ (green), $\mathrm{VBr}_{3}$ (purple) and $\mathrm{Vl}_{3}$ (red) at $\mathrm{C} / 40$ alternating between 30 min galvanostatic and 2 hours of relaxation. b) Electronegativity of the halide ligands according to the Pauling scale and impacts on the orbital structure. c) Operando evolution of the V K-edge spectra measured by X-ray absorption spectroscopy during the discharge of $\mathrm{VCl}_{3}$ at $\mathrm{C} / 20$. $\mathbf{d}$ ) Comparison of the spectra recorded at the open circuit voltage (blue), end at the end of discharge (green) with $\mathrm{VCl}_{2}$ reference material (red).

To gain a deeper understanding of the kinetics of the redox process taking place during intercalation, galvanostatic intermittent titration technique (GITT) was performed. Interestingly, the quasi-equilibrium 
path is almost identical for the three halides. Two discharge plateaus are observed, only differing in their potentials which correlate nicely with the ligand electronegativity (the more electronegative the halide, the higher the potential, Figure $\mathbf{3} \mathbf{a}, \mathbf{b}$ ). This result suggests that the electrochemical trace is solely related to the redox potential of the $\mathrm{V}(\mathrm{III}) \mathrm{N}(\mathrm{II})$ couple. To validate this charge compensation mechanism, operando X-ray absorption spectroscopy was performed on $\mathrm{VCl}_{3}$ (Figure $\mathbf{3} \mathbf{c}$ ). During the discharge, a shift of the $\mathrm{V} \mathrm{K}$-edge position to lower energy (Figure $\mathbf{3} \mathbf{c}$ ) reveals a shift from V(III) (pristine) to V(II) (Figure $\mathbf{3} \mathbf{d}$ ). The overall evolution during the discharge can be fully described using three principal components: the pristine phase, the end of discharge phase and an intermediate phase (Supplementary Figures 9, 10 and 11), confirming the existence of an intermediate phase as previously observed during operando synchrotron XRD.

\section{Rationale for the decreased solubility of halides in superconcentrated electrolytes}

In summary, we directly proved that superconcentrated electrolytes can be used to explore new intercalation compounds and synthesize novel phases for chemistries previously disregarded as highly soluble in liquid electrolytes. To rationalize this effect, the solubility of $\mathrm{VCl}_{3}$ was measured at different concentrations of LiFSI as supporting salt in DMC by inductively coupled plasma mass spectrometry (ICPMS). The results, shown in Figure $\mathbf{4}$ a, reveal an initial increase of the vanadium solubility as a function of the LiFSI concentration followed by a drastic decrease when LiFSI concentration is greater than $1 \mathrm{M}$, reaching values as low as few mM for the LiFSI 5 M superconcentrated electrolyte. Furthermore, vanadium chloride powder was mixed with $5 \mathrm{M} \mathrm{LiFSI}$ in DMC solutions for 3 days at $55^{\circ} \mathrm{C}$ and $85^{\circ} \mathrm{C}$, before allowing the solutions to rest at room temperature and monitoring the amount of dissolved vanadium. Since the measured concentrations were extremely close to the ones obtained at room temperature $\left(\mathrm{Cv}_{V}(R T)=6.3 \mathrm{mM}, \mathrm{cv}_{V}\left(55^{\circ} \mathrm{C}\right)\right.$ $=7.6 \mathrm{mM}$ and $\left.\mathrm{c}_{\mathrm{v}}\left(85^{\circ} \mathrm{C}\right)=5.5 \mathrm{mM}\right)$, any scattering of the results due to a kinetic hindering of the powder dissolution can be discarded to explain the aforementioned trend. Moreover, similar results were obtained for $\mathrm{VI}_{3}$ and $\mathrm{VBr}_{3}$ (Supplementary Figure 12). Thus, this peculiar bell shape reflects a thermodynamicallydriven phenomenon. 
a

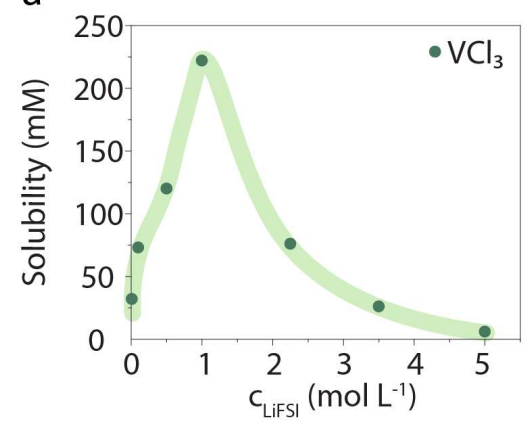

b

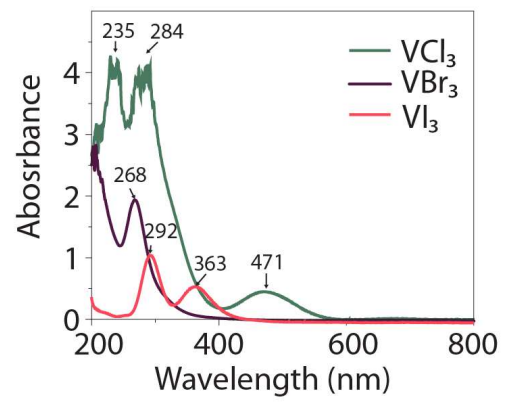

$C$

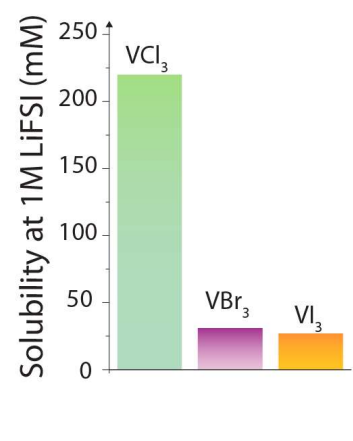

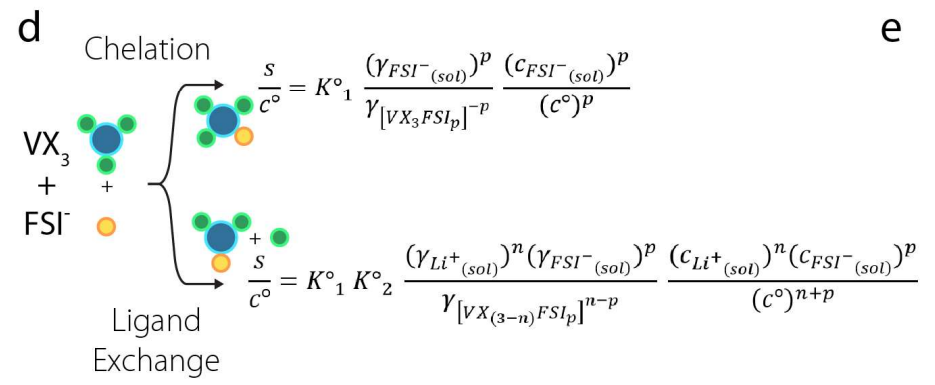

e

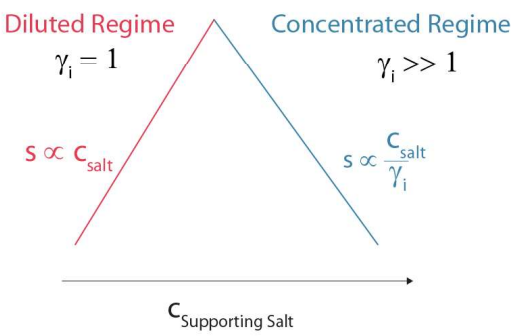

Figure 4. Superconcentrated electrolytes thermodynamically prevent vanadium halides dissolution. Evolution of $\mathrm{VCl}_{3}$ solubility measured by ICP-MS LiFSI/DMC electrolytes as a function of the LiFSI concentration. b) UV-vis spectra for 1 M LiFSI in DMC solutions saturated in $\mathrm{VCl}_{3}$ (green), $\mathrm{VBr}_{3}$ (purple), and $\mathrm{VI}_{3}$ (red). The spectrum for $\mathrm{VCl}_{3}$ shows some saturation, which is related to the high vanadium concentration, even when measured in a $0.1 \mathrm{~mm}$ optical pathway cuvette. c) Solubilities measured for $\mathrm{VCl}_{3}$ (green), $\mathrm{VBr}_{3}$ (purple) and $\mathrm{VI}_{3}$ (orange) by ICP-MS in a $1 \mathrm{M} \mathrm{LiFSI}$ in DMC electrolyte. d) Expression of the vanadium halide solubility as a function of the dissolution mechanism (chelation or ligand exchange) and e) schematic representation of the thermodynamics laws governing the transition metal solubility as a function of the supporting salt concentration.

To derive the equilibrium law that governs the solubility of vanadium at different supporting salt concentrations, we first need to understand the nature of the dissolution reaction by identifying the chemical environment of the vanadium cations dissolved in solution. Visually, every $1 \mathrm{M}$ LiFSI solution saturated with $\mathrm{VX}_{3}$ exhibits a pronounced coloration, as evidenced by the presence of absorption peaks in their UV-vis spectra (Figure $\mathbf{4} \mathbf{b}$ ). Interestingly, the wavelengths of the absorption peaks are halidedependent, suggesting the formation of a vanadium-halide complex in solution. Moreover, the initial increase of the solubility with LiFSI concentration (at concentrations below $1 \mathrm{M}$ ) advocates for the participation of the salt anion in the formation of this complex. Such observations can be rationalized by the formation of adducts in solution in the form of $\left[\mathrm{VX}_{3-\mathrm{n}} \mathrm{FSI}_{\mathrm{p}}\right]^{\mathrm{n}-\mathrm{p}}$, through a chelation or a ligand exchange mechanism, as proposed below: 


$$
V X_{3_{(\text {crist })}}+p F S I_{(\text {sol })}^{-}=\left[V X_{(3-n)} F S I_{p}\right]^{n-p}+n X^{-}
$$

where $n$ and $p$ are integers with $0 \leqslant n \leqslant 3$ and $p>0$ ( $n=0$ for the chelation mechanism).

Moreover, the solubility of $\mathrm{LiCl}$ is very low in pure DMC $(1.3 \mathrm{mM}) .{ }^{23}$ Hence, since the $\mathrm{VCl}$ s solubility in $1 \mathrm{M} \mathrm{LiFSI}$ electrolyte is around 100 times greater than this value, $\mathrm{Cl}^{-}$ions must be generated from the $\mathrm{VCl}_{3}$ dissolution and should undergo an almost complete re-precipitation:

$$
\mathrm{Li}^{+}{ }_{(\mathrm{sol})}+\mathrm{Cl}^{-}{ }_{(\mathrm{sol})}=\mathrm{LiCl}_{(\mathrm{crist})}\left(\mathrm{K}_{2}^{\circ} \gg 1\right)
$$

This second step, which can hardly be observed due to the very limited amount of LiCl precipitated (see discussion in the SI), can drastically shift the first equilibrium towards the formation of the product, as expressed by the equation below:

$$
V X_{3_{(c r i s t)}}+p F I^{-}{ }_{(s o l)}+n L^{+}{ }_{(s o l)}=\left[V X_{(3-n)} F S I_{p}\right]^{n-p}+n \operatorname{LiX}_{(\text {crist })}\left(K=K_{1}^{\circ} K_{2}^{\circ}\right)
$$

At the thermodynamic equilibrium, the Guldberg and Waage law of mass action gives:

$$
K=K^{\circ}{ }_{1} K_{2}^{\circ}=\frac{a_{\left[V X_{(3-n)} F S I_{p}\right]^{n-p}}}{\left(a_{L i^{+}(s o l)}\right)^{n}\left(a_{F S I^{-}(\text {sol })}\right)^{p}}
$$

Thus, considering the activity of a solute as the product of its concentration cand its activity coefficient $\gamma$, divided by the standard concentration $\left(c^{\circ}=1 \mathrm{~mol} \mathrm{~L}^{-1}\right)$, the solubility $s$ of the $\mathrm{VX}_{3}$ salt can be expressed by the following equation:

$$
\frac{s}{c^{\circ}}=K_{1}^{\circ} K_{2}^{\circ} \frac{\left(\gamma_{L i^{+}}{ }_{(s o l)}\right)^{n}\left(\gamma_{F S I^{-}}{ }_{(s o l)}\right)^{p}}{\gamma_{\left[V X_{(3-n)} F S I_{p}\right]^{n-p}}} \frac{\left(c_{L i^{+}}{ }_{(s o l)}\right)^{n}\left(c_{F S I^{-}}{ }_{(s o l)}\right)^{p}}{\left(c^{\circ}\right)^{n+p}}
$$

Since the solubility of $\mathrm{LiBr}\left(\mathrm{S}_{\mathrm{LBBr}}\right)$ and $\mathrm{Lil}\left(\mathrm{S}_{\mathrm{Li}}\right)$ are expected to be greater than the one of $\mathrm{LiCl}$ (e.g. $\mathrm{S}_{\mathrm{LBBr}}=4 \mathrm{mM}$ in $\mathrm{DMC}$ at $25^{\circ} \mathrm{C}$, 3 times that of $\left.\mathrm{LiCl}\right)^{23}$, one would expect $\mathrm{K}_{2}^{\circ}(\mathrm{Cl})>\mathrm{K}_{2}^{\circ}(\mathrm{Br})>\mathrm{K}_{2}^{\circ}(\mathrm{I})$, which is consistent with the trend observed for the solubilities of the $\mathrm{VX}_{3}$ compounds that follows $\mathrm{VCl}_{3}>\mathrm{VBr}_{3}>\mathrm{VI}_{3}$ (Figure 4 c). For a 
chelation mechanism, $\mathrm{K}_{2}^{\circ}=1$ and the solubilities of the different vanadium halides are expected to vary less than for the ligand-exchange mechanism. Hence, our experimental observations point towards dissolution through a ligand-exchange mechanism (see SI for a deeper discussion). Neglecting a variation of the activity coefficients, hypothesis which usually holds for low salt concentrations, ${ }^{24}$ the $V X_{3}$ solubility should thus increase with the LiFSI concentration, in agreement with our experimental observations made for concentrations < $1 \mathrm{M}$ (Figure 4 a). However, such explanation does not hold anymore at high ionic strength, regime in which the activity coefficients are known to largely deviate from unity. ${ }^{24}$ First, as expressed in equation (5), a large decrease of $\gamma_{\mathrm{Li}^{+}}$and $\gamma_{\mathrm{Cl}^{-}}$could potentially explain the lowering of the $\mathrm{VX}{ }_{3}$ solubility. Nevertheless, as suggested in a recent theoretical work, the activity coefficients of single ions are more likely to be greater at high salt concentrations. ${ }^{24}$ Hence, the decreased solubility is rather explained by a large increase of $\gamma_{\left[V X_{(3-n)} F S I_{p}\right]^{n-p}}$ which, owing to the interaction of the vanadium adduct with surrounding solvent molecules, would require developing a refined model to accurately account for the variation of its activity coefficient with the ionic strength. We should further emphasize that independently on the dissolution mechanism (ligand-exchange or chelation), the $V X_{3}$ solubilities are expected to vary in a similar manner depending on the LiFSI concentration (Figure $4 \mathbf{d}$ ).

Our study provides the rationalization for the observed bell shape behavior: the initial increase of the solubility originates from the increased concentration of FSI anions, while the decrease observed in the concentrated regime arises from a large deviation from unity of the transition-metal complex activity coefficient value (Figure 4e). More importantly application-wise, the solubility of the fully discharged $\mathrm{LiVCl}_{3}$ phase shows similar bell shape (Supplementary Figure 13), albeit the solubility is found lower than for $\mathrm{VCl}_{3}$ which ensures a good resistance to dissolution for the material even upon reduction. However, we note that upon long cycling a severe capacity decay is observed and can be attributed to vanadium dissolution accumulated over time (Supplementary Figure 14). Obviously, future work ranges in better tuning the delicate electrolyte-solvent balance to improve the capacity retention both at RT and $55^{\circ} \mathrm{C}$. In conclusion, 
these findings confirm that the fundamental framework developed in this work captures every stage of lithiation and can be transposed to the study of other intercalation compounds.

Overall, we established the use of superconcentrated electrolyte as a platform to discover new families of materials for $\mathrm{Li}^{+}$intercalation. Driven by the broad interest for their physical properties, we (reversibly) intercalated $\mathrm{Li}^{+}$into layered vanadium halides to form new layered phases, the impact of which goes beyond the sole development of intercalation electrodes for secondary batteries. Indeed, preliminary nuclear magnetic resonance (NMR) results (Supplementary Figure 15) suggest a fast exchange of $\mathrm{Li}^{+}$cations between different crystallographic sites, which does not come as a total surprise owing to the ongoing interests for halides as solid state ionic conductors. Moreover, these layered halides have been widely investigated for their promising magnetic properties, and preliminary magnetic susceptibility measurements show that $\mathrm{Li}^{+}$intercalation can be used to tune their magnetic structure. Aside from unlocking the synthesis of new layered structures, our study also lays the fundamental background to comprehend a so far ill-understood effect related to the use of superconcentrated electrolytes, i.e. the decreased solubility of active material. Indeed, we demonstrate that the low solubility of transition metal compounds in superconcentrated electrolytes originates from a shift of the solubility equilibrium, i.e. from a thermodynamic effect rather than a kinetics one, which also applies to current collectors whose stability in presence of corrosive anions (e.g. FSI, TFSI) was observed to increase in superconcentrated electrolytes. In general, our study highlights the critical need for solid-state chemists to include knowledge about the physical chemistry of liquids when developing novel intercalation compounds. Evidently, the future success of such explorative and collaborative work will rely on answering fundamental questions regarding equilibrium and ion activities in this novel class of electrolytes. 


\section{Material and methods}

Synthesis

$\mathrm{VBr}_{3}$ and $\mathrm{Vl}_{3}$ were grown in evacuated quartz sealed tubes by reacting elementary vanadium (vanadium powder, -325 mesh 99.5\% - Alfa Aesar), iodine (99+\%, Alfa Aesar) and purified bromine (Sigma-Aldrich) with a slight excess of halide. Because bromine is a liquid, the tube was place in a liquid nitrogen bath to freeze bromine before being evacuated and quickly flame-sealed. The tubes were placed in a tubular oven, the extremity containing the reactants being heated at $400^{\circ} \mathrm{C}$ for $\mathrm{VBr}_{3}$ and $450^{\circ} \mathrm{C}$ for $\mathrm{Vl}_{3}$ while the other extremity was placed at almost room temperature, as reported elsewhere. ${ }^{1,2}$ After a $72 \mathrm{~h}$ synthesis, large crystals were collected at the cold extremity of the tube, placed in a Schlenk tube and further heated at $200^{\circ} \mathrm{C}$ for few hours under vacuum (10-2 mbar) to eliminate the excess bromine or iodine traces. $\mathrm{VCl}_{3}$ (97\%, Sigma-Aldrich) was used as received. Lithiated phases were obtained by adding the $V X_{3}$ phases in a 3 times excess of $n$ butyllithium solution (2.5 $\mathrm{M}$ in hexane, Sigma Aldrich) and stirring the suspension for at least 1 hour. The solution was further centrifuged and the collected powder was washed 3 times with hexane before drying under vacuum in the glovebox antechamber.

The $\beta$ - $\mathrm{Li}_{3} \mathrm{PS}_{4}$ solid electrolyte was obtained in our laboratory via a THF-mediated route proposed by Liu et al. ${ }^{25}$ and with a room temperature ionic conductivity of $\sigma_{\mathrm{RT}}=0.21 \mathrm{mS} / \mathrm{cm}$. On the other hand, commercial $\mathrm{Li}_{6} \mathrm{PS}_{5} \mathrm{Cl}$ electrolyte was used (NEI), having an ionic conductivity of $\sigma_{\mathrm{RT}}=3.8 \mathrm{mS} / \mathrm{cm}$.

\section{Electrochemical measurements}

\section{Liquid electrolytes}

All the electrochemical experiments were carried out in an Ar-filled glovebox. All the materials tested were mixed with conductive carbon super-P in an active-material/carbon ratio of $7 / 3$. For all the experiments using a liquid electrolyte (LP30 1 M LiPF6 dissolved in 1:1 v/v ethylene carbonate/dimethyl carbonate, 
Solvionic or LiFSI $5 \mathrm{M}$ in dimethyl carbonate, Solvionic) the as prepared composite were tested in a coin-cell configuration versus a Li metal negative electrode separated by one Whatman glass fiber separator, soaked with $\sim 100 \mu \mathrm{L}$ of electrolyte. The mass of composite loaded in the coin cells was comprised between 3 and $5 \mathrm{mg}$. The cells were cycled on VMP or MPG potentiostats from BioLogic at room temperature, except for the GITT for which the cells we placed in a $25^{\circ} \mathrm{C}$ oven.

\section{Solid-state batteries}

Both anode and cathode composites were prepared by hand grinding the components in the proportions mentioned below with mortar and pestle within an Ar-filled glovebox. The electrochemical testing of the $\mathrm{VX}_{3}$ compounds $(\mathrm{X}=\mathrm{Cl}, \mathrm{Br}, \mathrm{I})$ in all-solid state configuration was conducted in a three-pieced homemade cell consisting in two stainless steel pistons which are inserted into a PEI body (Supplementary Figure 16). The cell is closed by means of six axial screws which also provide the pressure required for correct operation. Additionally, a ferrule-cone pair is also integrated in each piston making the setup airtight.

For the battery assembling $35 \mathrm{mg}$ of $\mathrm{Li}_{6} \mathrm{PS}_{5} \mathrm{Cl}$ electrolyte were firstly loaded into the cell and cold pressed at $200 \mathrm{~kg} / \mathrm{cm}^{2}$ for 1 minute. Next, 10 to 12 mg/cm² of catholyte (VX: $\left(\beta-\mathrm{Li}_{3} P S_{4}: V G C F\right.$, 65:30:5 wt.\%) were evenly spread onto one side of the compressed solid electrolyte and a pressure of $1000 \mathrm{~kg} / \mathrm{cm}^{2}$ was applied during 1 minute. Lastly, 35 mg of anode composite ( $\mathrm{Li}_{0.8} \mathrm{In}$ : $\left.\mathrm{Li}_{6} \mathrm{PS}_{5} \mathrm{Cl}, 60: 40 \mathrm{wt} . \%\right)$ were added onto the opposite side and the whole stack was densified at $4000 \mathrm{~kg} / \mathrm{cm}^{2}$ for $15 \mathrm{Min}$. The fully assembled cell was later closed with a torque key applying $2.3 \mathrm{Nm}$ torque in each screw, which yields an internal pressure of $\sim 1000 \mathrm{~kg} / \mathrm{cm}^{2}$. Galvanostatic cycling of the cells was carried out at room temperature, in the voltage range at C/10 using the VMP3 electrochemical workstation by Bio-Logic Science Instruments SAS. 


\section{Materials characterization}

Ex Situ synchrotron X-ray diffraction (SXRD) patterns were collected on the BL04-MSPD beamline of the ALBA synchrotron (Barcelona area, Spain) at wavelength $\lambda=0.41378 \AA$ using the Position Sensitive Detector MYTHEN. Powder samples were filled in $0.6 \mathrm{~mm}$ diameter borosilicate capillaries inside an Ar-atmosphere glovebox and subsequently flame-sealed. Operando measurements were carried out in transmission mode using dedicated coin cells ${ }^{26}$ assembled under argon filled glovebox and mounted on an ALBA designed 4 samples changer. Constant wavelength $(\lambda=1.622 \AA)$ neutron powder diffraction (NPD) data were collected for $\operatorname{LiVX}_{3}(X=C l, B r, I)$ at room temperature using the ECHIDNA high angular resolution powder diffractometer installed at the OPAL research reactor (Lucas Heights, Australia). ${ }^{27}$ To prevent reaction of the samples with ambient atmosphere, the samples were loaded into $9 \mathrm{~mm}$ diameter cylindrical vanadium cans in Ar-filled glove box and sealed with In seals. All diffraction patterns were refined using the FullProf program.

The scanning electron microscopy images were measured on a FEl Magellan scanning electron microscope equipped with an Energy dispersive X-ray spectroscopy (EDX) Oxford Instrument detector. EDX measurements were carried out using an acceleration voltage of $10 \mathrm{kV}$. The Li metal anode samples were collected from cycled cells, washed with dimethyl carbonate and sealed in an air tight container. The transfer from the container to the microscope vacuum chamber were realized rapidly ( $\sim 10$ s) to minimize air exposure.

\section{X-Ray Absorption Spectroscopy}

Synchrotron X-ray absorption spectroscopy was performed at the vanadium K-edge at the ROCK beamline of the SOLEIL synchrotron facility (Saint-Aubin, France). ${ }^{28}$ The Si(111) quick-XAS monochromator with an oscillation frequency of $2 \mathrm{~Hz}$ was employed to select the incident photons energy. The spectra were collected in transmission using three gas ionization chambers in series as detectors. A vanadium metal foil was placed between the second and the third ionization chambers to ensure the energy calibration. An 
average of 900 scans per spectrum (corresponding to 15 minutes of acquisition time for one merged XAS spectrum) was recorded to ensure the reproducibility and to increase the signal-to-noise ratio. The $\mathrm{VCl}_{2}$ and $\mathrm{VCl}_{3}$ reference samples were prepared by mixing uniformly the active material with carbon, then pressed into pellets of $10 \mathrm{~mm}$ in diameter. For the operando measurements, a self-standing electrode was prepared by mixing $\mathrm{VCl}_{3}$ active material with carbon black and polytetrafluoroethylene (PTFE) in the ratio of 20:70:10 (by wt.\%). The electrode was placed in the in situ electrochemical cell29 and cycled against metallic Li using a Celgard membrane as separator and $\sim 100 \mu \mathrm{L}$ of $5 \mathrm{M} \mathrm{LiFSI}$ in dimethyl carbonate (DMC) as electrolyte. Then, the in situ cell was discharged from the OCV (3.025 V) to $2.5 \mathrm{~V} \mathrm{vs} \mathrm{Li+/Li} \mathrm{at} \mathrm{a} \mathrm{C-rate} \mathrm{of} \mathrm{C/20} \mathrm{(corresponding} \mathrm{to}$ 1 mole of $\mathrm{Li}^{+}$inserted in 20 hours), and a XAS spectrum was recorded every 15 minutes. The details for the principal component analysis are given in the SI.

\section{Solubility measurements}

To ensure that the concentrations of vanadium measured in the electrolytes match the thermodynamic limit of solubility for $\mathrm{VX}_{3}$ materials, a large excess of powder ( 100-500 mg, depending on the material) was added in $1 \mathrm{~mL}$ of organic electrolyte and stirred for 3 days at room temperature in a Ar-filled glovebox. The as prepared solutions were centrifuged (6 000 rpm, 1 hour), and the supernatant was collected and further filtered on $0.2 \mu \mathrm{m}$ pore size PTFE syringe filter in an Ar-filled glovebox. Outside of the glovebox, the as prepared solutions were diluted 1000 or 10000 times in a $2 \mathrm{~W}_{\%} \mathrm{HNO}_{3}$ (prepared from $\mathrm{HNO}_{3}$ 99.999\% metal basis Alfa Aesar and Mili-Q ultrapure water) to reach final vanadium concentrations below $1 \mathrm{ppm}$. The vanadium concentration in the as prepared aqueous solutions were measured by ICP-MS (Nexion 2000 Perkin Elmer) using a calibration curve obtained by diluting a vanadium standard solution for ICP-MS (TraceCERT, 1 mg/L V in nitric acid, Sigma Aldrich). 
UV-vis characterization

The organic solutions were placed in a $0.1 \mathrm{~mm}$ optical pathway cuvette (Helma) and their UV-vis spectra was measured with a UV5 Bio Mettler Toledo spectrophotometer using a 1 M LiFSI in dimethyl carbonate blank solution.

\section{Acknowledgements}

I. Aguilar and V. Meunier are gratefully acknowledged for their help with ICP measurements. P. Lemaire is gratefully acknowledged for the SEM/EDX measurements. N.D. acknowledges the Ecole Normale Supérieure for his PhD. Scholarship. T.M. acknowledges the Ecole Normale Supérieure Paris-Saclay for his PhD scholarship. A.G. acknowledge support from the Region lle-de-France in the framework of DIM ResPore for the ICP-MS purchase. A.G. acknowledges financial support from the ANR MIDWAY (Project ID ANR-17-CE050008). N.D., T.M., G.R., F.M., A.I., B.P., M.D., J.-M.T., A.G. thank the French National Research Agency for its support through the Labex STORE-EX project (ANR-10LABX-76-01). Use of the 11-BM mail service of the APS at Argonne National Laboratory was supported by the US Department of Energy under contract No. DEAC02-06CH1 1357 and is gratefully acknowledged. XAS experiments were performed on the ROCK beamline

(financed by ANR- 10-EQPX-45) at SOLEIL Synchrotron, France, under proposal \#20200810. ALBA experiments were performed through academic proposal 2020024152

Author contributions

N.D., T.M., J-M.T. and A.G. designed the research. T.M., N.D., and J-M.T. carried out the synthesis. T.M., F.F., and M.A. performed the structural characterizations, further analyzed with the help of G.R. N.D. conducted the 
electrochemical and the solubility measurements. F.M. assembled and tested the solid-state batteries. B.P. and M.D. conducted and analyzed the NMR experiments. A.I. performed the XAS operando experiments and analyzed the data. All the authors discussed the scientific results and contributed to the writing of the manuscript.

Competing interests

The authors declare no competing interests 


\section{References}

1. Kong, T., Guo, S., Ni, D. \& Cava, R. J. Crystal structure and magnetic properties of the layered van der Waals compound $\mathrm{VBr}_{3}$. Phys. Rev. Materials 3, 084419 (2019).

2. Kong, T. et al. $\mathrm{Vl}_{3}$ —a New Layered Ferromagnetic Semiconductor. Advanced Materials 31, 1808074 (2019).

3. Song, T. et al. Switching 2D magnetic states via pressure tuning of layer stacking. Nature Materials 18, 1298-1302 (2019).

4. Berthelot, R., Carlier, D. \& Delmas, C. Electrochemical investigation of the $\mathrm{P} 2-\mathrm{NaxCoO}_{2}$ phase diagram. Nature Mater 10, 74-80 (2011).

5. Skyllas-Kazacos, M., Cao, L., Kazacos, M., Kausar, N. \& Mousa, A. Vanadium Electrolyte Studies for the Vanadium Redox Battery-A Review. ChemSusChem 9, 1521-1543 (2016).

6. Dey, A. N. \& Sullivan, B. P. The Electrochemical Decomposition of Propylene Carbonate on Graphite. J. Electrochem. Soc. 117, 222 (1970).

7. Fong, R. Studies of Lithium Intercalation into Carbons Using Nonaqueous Electrochemical Cells. J. Electrochem. Soc. 137, 5 (1990).

8. Jeong, S.-K., Inaba, M., Iriyama, Y., Abe, T. \& Ogumi, Z. Electrochemical Intercalation of Lithium lon within Graphite from Propylene Carbonate Solutions. Electrochem. Solid-State Lett. 6, A13 (2002).

9. Yamada, Y., Takazawa, Y., Miyazaki, K. \& Abe, T. Electrochemical Lithium Intercalation into Graphite in Dimethyl Sulfoxide-Based Electrolytes: Effect of Solvation Structure of Lithium lon. J. Phys. Chem. C114, 11680-11685 (2010).

10. Yamada, Y. et al. General Observation of Lithium Intercalation into Graphite in Ethylene-Carbonate-Free Superconcentrated Electrolytes. ACS Appl. Mater. Interfaces 6, 10892-10899 (2014).

11.Yamada, Y. et al. Unusual Stability of Acetonitrile-Based Superconcentrated Electrolytes for FastCharging Lithium-Ion Batteries. Journal of the American Chemical Society 136, 5039-5046 (2014). 
12.Suo, L. et al. "Water-in-salt" electrolyte enables high-voltage aqueous lithium-ion chemistries. Science 350, 938-943 (2015).

13.Wang, J. et al. Superconcentrated electrolytes for a high-voltage lithium-ion battery. Nat. Commun. 7, 12032 (2016).

14.Yamada, Y. et al. Hydrate-melt electrolytes for high-energy-density aqueous batteries. Nat. Energy 1, 16129 (2016).

15.Wang, J. et al. Fire-extinguishing organic electrolytes for safe batteries. Nat. Energy 3, 22-29 (2018).

16.Yamada, Y., Wang, J., Ko, S., Watanabe, E. \& Yamada, A. Advances and issues in developing saltconcentrated battery electrolytes. Nat. Energy (2019) doi:10.1038/s41560-019-0336-z.

17.Yue, J. et al. Interface Concentrated-Confinement Suppressing Cathode Dissolution in Water-in-Salt Electrolyte. Adv. Energy Mater. 2000665 (2020) doi:10.1002/aenm.202000665.

18.Sun, D., Okubo, M. \& Yamada, A. Optimal water concentration for aqueous $\mathrm{Li}^{+}$intercalation in vanadyl phosphate. Chem. Sci. 10.1039.D0SC04647G (2021) doi:10.1039/D0SC04647G.

19.Mendiboure, A., Delmas, C. \& Hagenmuller, P. Electrochemical intercalation and deintercalation of $\mathrm{Na}_{x} \mathrm{MnO}_{2}$ bronzes. Journal of Solid State Chemistry 57, 323-331 (1985).

20.Dubouis, N. et al. Chasing Aqueous Biphasic Systems from Simple Salts by Exploring the LiTFSI/LiCl/ $\mathrm{H}_{2} \mathrm{O}$ Phase Diagram. ACS Cent. Sci. 5, 640-643 (2019).

21.Serva, A., Dubouis, N., Grimaud, A. \& Salanne, M. Confining Water in lonic and Organic Solvents to Tune Its Adsorption and Reactivity at Electrified Interfaces. Acc. Chem. Res. (2021) doi:10.1021/acs.accounts.0c00795.

22.Soubeyroux, J. L., Cros, C., Gang, W., Kanno, R. \& Pouchard, M. Neutron diffraction investigation of the cationic distribution in the structure of the spinel-type solid solutions $\mathrm{Li}_{2-2 x} \mathrm{M}_{1+x} \mathrm{Cl}_{4}(\mathrm{M}=\mathrm{Mg}, \mathrm{V})$ : Correlation with the ionic conductivity and NMR data. Solid State Ionics 15, 293-300 (1985).

23.Xin, N., Sun, Y., He, M., Radke, C. J. \& Prausnitz, J. M. Solubilities of six lithium salts in five non-aqueous solvents and in a few of their binary mixtures. Fluid Phase Equilibria 461, 1-7 (2018). 
24.McEldrew, M., Goodwin, Z. A. H., Bi, S., Bazant, M. Z. \& Kornyshev, A. A. Theory of ion aggregation and gelation in super-concentrated electrolytes. J. Chem. Phys. 152, 234506 (2020).

25.Liu, Z. et al. Anomalous High lonic Conductivity of Nanoporous $\beta$ - $\mathrm{Li}_{3} \mathrm{PS}_{4}$. $20-23$ (2013) doi:10.1021/ja3110895.

26. Herklotz, M. et al. A novel high-throughput setup for in situ powder diffraction on coin cell batteries. J App/ Crystallogr 49, 340-345 (2016).

27.Avdeev, M. \& Hester, J. R. ECHIDNA: a decade of high-resolution neutron powder diffraction at OPAL. J App/ Cryst 51, 1597-1604 (2018).

28.Briois, V. et al. ROCK: the new Quick-EXAFS beamline at SOLEIL. J. Phys.: Conf. Ser. 712, 012149 (2016). 29.Leriche, J. B. et al. An Electrochemical Cell for Operando Study of Lithium Batteries Using Synchrotron Radiation. J. Electrochem. Soc. 157, A606 (2010). 